\title{
Bioimpedância para avaliação da composição corporal: uma proposta didático-experimental para estudantes da área da saúde
}

Bioelectrical Impedance for body composition evaluation: an experimental didactic proposal for health sciences students

\author{
Marcelo Mariano da Silva*1@, Raquel Santos Marques de Carvalho ${ }^{1}$, Marcelo Baptista de Freitas ${ }^{1}$ \\ ${ }^{1}$ Universidade Federal de São Paulo, Departamento de Biofísica, Escola Paulista de Medicina, São Paulo, SP, Brasil
}

Recebido em 25 de Setembro, 2018. Revisado em 24 de Outubro, 2018. Aceito em 25 de Outubro, 2018.

\begin{abstract}
Este artigo apresenta um experimento de bioimpedância voltado para estudantes da área da saúde e elaborado a partir de instrumentação tradicional encontrada em laboratórios de ensino de cursos de graduação. Essa proposta permite a introdução de grandezas físicas e conceitos básicos de circuitos elétricos através de um recorte interdisciplinar de medida de impedância do corpo humano e avaliação corporal. Sugere-se que o uso didático do experimento seja feito em etapas: (a) familiarização com o uso do osciloscópio e demais equipamentos, montagem do circuito equivalente para simular as medidas de bioimpedância e comparação das grandezas medidas e calculadas; (b) condução de medidas "in vivo"; (c) cálculo das variáveis de interesse clínico a partir dos valores determinados experimentalmente de $\mathrm{R}$ e $\chi_{c}$ a $50 \mathrm{kHz}$ e dos parâmetros antropométricos medidos. Concluiu-se que a operacionalização do experimento para fins didáticos é viável e seu emprego adequado para que os estudantes ascendam a uma melhor compreensão da medida de bioimpedância, tornando-se críticos em relação ao emprego de equipamentos comerciais na prática clínica. A exposição prática e conceitual usada reforça a importância de se re-examinar o propósito do conhecimento tradicional disciplinar na solução de problemas da área da saúde.

Palavras-chave: Bioimpedância, Conceitos Básicos, Interdisciplinaridade, Princípios Físicos, Laboratório de Ensino.
\end{abstract}

This article presents a bioelectrical impedance experiment for health science students using the traditional instrumentation available in undergraduate laboratories. Such proposal allows the study of electrical circuits physical principles and basic concepts through an interdisciplinary approach of bioelectrical impedance measurements and body evaluation. The didactical use of the experiment can be done in steps: (a) familiarization with an oscilloscope and other equipment. The equivalent circuit must be built to simulate the bioelectrical impedance and also do the comparison between the physical and the calculated quantities; (b) implementation of "in vivo" measurements; (c) computation of clinical variables from anthropometric parameters and experimental values of $\mathrm{R}$ and $\chi_{c}$ at $50 \mathrm{kHz}$. The conclusion is that the proposal is viable and its usage is appropriate for a critical thinking of bioelectrical impedance comprehension including the use of commercial equipment in clinical practice. The practical and conceptual approach presented reinforces the importance of re-examine the purpose of the traditional disciplinary knowledge to deal with health science challenges.

Keywords: Bioimpedance, Basic Concepts, Interdisciplinarity, Physical Principles, Laboratory Learning.

\section{Introdução}

Nos cursos de graduação da área da saúde é comum a abordagem de conceitos da física sem contextualização e tratamento interdisciplinar apropriados, dificultando que novas perspectivas sobre problemas práticos sejam alcançadas por parte dos alunos. Por isso, construir um ambiente de ensino-aprendizagem com transversalidade e interdisciplinaridade, ou seja, que implique na articulação entre conhecimentos sistematizados e questões da vida real, é essencial para significar o conhecimento e desenvolver habilidades que preparem "os professores

*Endereço de correspondência: marcelo.mariano@unifesp.br para a manutenção desse ambiente e os alunos para a vida e para um ambiente de trabalho cada vez mais complexo, competitivo e exigente" 11. Nesse contexto, este artigo apresenta um experimento de bioimpedância voltado para estudantes da área da saúde e elaborado a partir de instrumentação tradicional encontrada em laboratórios de ensino de cursos de graduação. Trata-se de uma proposta didática que permite a introdução de grandezas físicas e conceitos básicos de circuitos elétricos corrente elétrica alternada $\left(I_{A C}\right)$, potencial elétrico eficaz $(V)$, resistência $(R)$, reatância capacitiva $\left(\chi_{c}\right)$, impedância $(Z)$, frequência $(f)$ e ângulo de fase $(\varphi)$ - através de um recorte interdisciplinar de medida de impedância 
do corpo humano e avaliação corporal. Concomitantemente possibilita-se que estudantes de cursos diferentes daqueles tradicionalmente classificados como de exatas ascendam a uma compreensão sobre bioimpedância que não é possível através de medições clínicas conduzidas em hospitais e centros especializados, onde os equipamentos são normalmente empregados como "caixas-pretas".

Na seção 2 é apresentada a fundamentação que permite o emprego da bioimpedância para avaliação da composição corporal, juntamente com os aspectos teóricos e práticos do circuito elétrico equivalente empregado no experimento proposto. Na seção 3 são discutidas as medidas realizadas em diferentes configurações neste circuito para simular o comportamento eletrofisiológico do corpo humano e os resultados de medidas "in vivo". As variáveis de interesse clínico são então calculadas a partir de um modelo estabelecido na literatura e uma breve discussão sobre suas limitações é apresentada. Por fim, a seção 4 traz a proposta propriamente dita de uso do experimento em laboratório didático, finalizando com as conclusões, na seção 5 .

\section{Experimento de Bioimpedância}

\subsection{Fundamentos}

A análise de impedância bioelétrica corporal (AIBC) serve para a determinação da composição corporal, ou seja, de parâmetros de interesse clínico como massa de gordura corporal (MG), massa livre de gordura (MLG), massa de água intracelular (AIC), massa de água extracelular (AEC) e a massa de água corporal total (ACT). Estas informações são importantes, por exemplo, na preparação física e avaliação de atletas [2] [3], na avaliação nutricional, no monitoramento de pacientes com doenças crônicas, nos acompanhamentos da sarcopenia [2], da tendência de obesidade populacional e males associados [4] e até mesmo no prognóstico de algumas doenças. Por se tratar de um procedimento amplamente difundido, o domínio dos fundamentos básicos que subsidiam esta técnica e a maneira como são realizadas as análises/avaliações são essenciais para a compreensão da AIBC pelos diferentes profissionais da área da saúde.

A AIBC consiste na medida da impedância que o corpo humano oferece à passagem de uma corrente alternada. Quando uma corrente elétrica flui pelo corpo, a condução se dá através dos fluidos extracelulares (AEC) e intracelulares (AIC) compostos principalmente de água com eletrólitos (íons de sais, ácidos e bases) que correspondem a aproximadamente $73 \%$ da massa livre de gordura (MLG). O restante da MLG (27\%) é composta pelas proteínas e componentes viscerais, além dos minerais ósseos. Os tecidos adiposos, ossos e o ar dos pulmões comportam-se como isolantes [5].

A resistência corporal a passagem de corrente elétrica tem portanto relação inversa com a massa de água corporal total (ACT), resultante da soma da AEC e AIC.
Além da resistência $(R)$ à passagem da corrente alternada pelos fluidos há também a resistência imposta pelas membranas celulares dos tecidos magros, as quais são constituídas por duas camadas opostas de moléculas fosfolipídicas. Estas possuem duas partes, uma apolar (longa cadeia de ácidos graxos) e outra polar (grupos fosfatos), que se comportam eletricamente como se fossem capacitores separando duas porções condutoras, uma externa à célula, formada pela água extracelular (AEC) e outra interna à célula composta pela água intracelular (AIC) (Figura 1).

Essa resistência oferecida pelas membranas à passagem de uma corrente alternada devido ao fato das mesmas se comportarem como capacitores denomina-se reatância capacitiva $\left(\chi_{c}\right)$. A impedância corporal ou bioimpedância $\left(Z_{c}\right)$ é a combinação da resistência $(R)$ dos fluidos e da reatância capacitiva $\left(\chi_{c}\right)$ das membranas, que pode ser calculada através da equação(1) [6].

$$
Z_{c}^{2}=R^{2}+\chi_{c}^{2}
$$
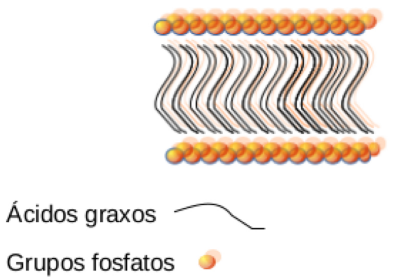

(a)

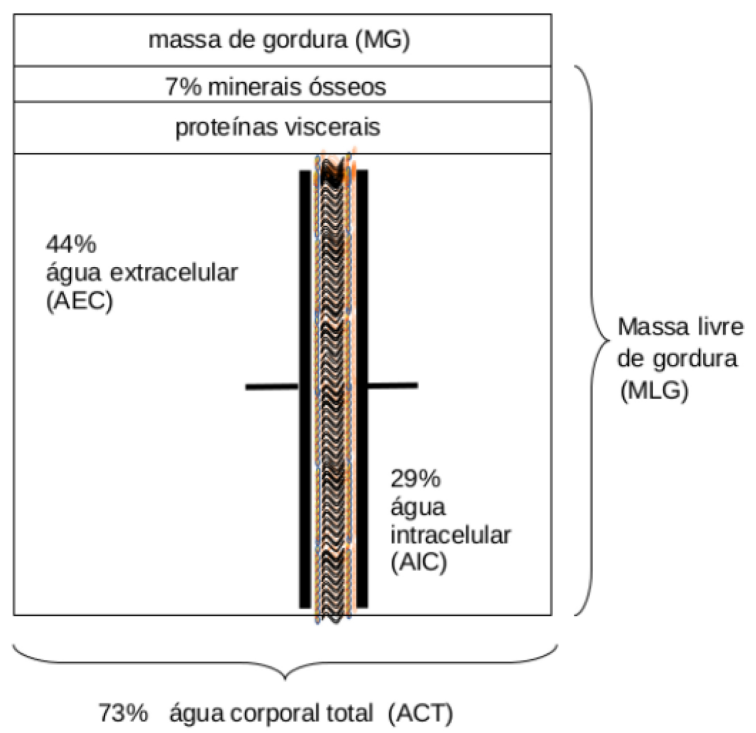

(b)

Figura 1: (a) A membrana é formada por dupla camada de moléculas fosfolipídicas, com as partes polares opostas, o que confere a ela propriedade elétrica semelhante a de um capacitor: duas camadas condutoras separadas por um material dielétrico, que são as cadeias de ácidos graxos; (b) Esquema representativo da composição corporal. 
Na equação (1), $R$ e $\chi_{c}$ podem representar valores ou funções dependendo do tipo de modelo eletrofisiológico que se atribui ao corpo humano. $\mathrm{Na}$ sua forma mais simples, assume-se que o organismo se comporta como uma associação em série de um resistor e um capacitor (Figura 2a) e $\chi_{c}$ é um valor que pode ser obtido da equação (2). Contudo outros modelos podem ser adotados (por exemplo os representados nas Figuras $2 \mathrm{~b}$ e $2 \mathrm{c})$ e nesses casos $R\left(R_{1}, R_{2}, \ldots R_{n}, C_{1}, C_{2}, \ldots C_{n}\right)$ e $\chi_{c}\left(R_{1}, R_{2}, \ldots R_{n}, C_{1}, C_{2}, \ldots C_{n}\right)$ passam a ser funções dos valores dos resistores e capacitores existentes nos circuitos escolhidos.

$$
\chi_{c}=\frac{1}{2 . \pi . \text { frequência.C }}
$$

O efeito capacitivo das membranas cria uma diferença de fase $(\Delta \varphi$ ou $\varphi$ por simplicidade) entre a tensão e a corrente aplicadas que pode ser calculada pela equação (3). Por exemplo, considerando-se que os valores de corrente e de tensão alternados sejam auferidos separadamente em cada um dos elementos da associação da Figura 2a, ou seja, no resistor e no capacitor, pode-se observar o comportamento da diferença de fase nas Figuras 3a e 3b.

No resistor há sincronia entre as variações de corrente e tensão, o que não ocorre no capacitor, onde existe uma diferença de fase $\varphi$.

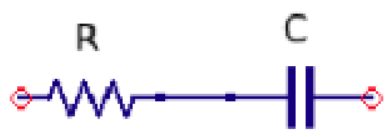

$2 a$

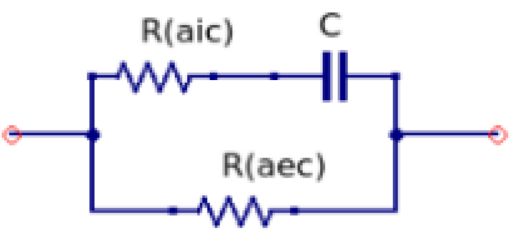

$2 b$

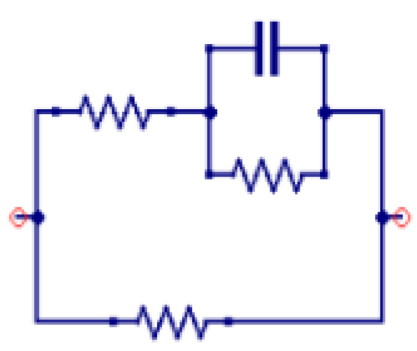

2c

Figura 2: (a) Associação série capacitor e resistor, modelo mais simples empregado em medidas que usam uma única frequência, geralmente de $50 \mathrm{kHz}$; (b) Exemplo de associação em paralelo, no qual são representadas a membrana (capacitor) e as resistências proporcionadas pela AIC e pela AEC [7]; (c) Associação em paralelo mais complexa que as anteriores [8].

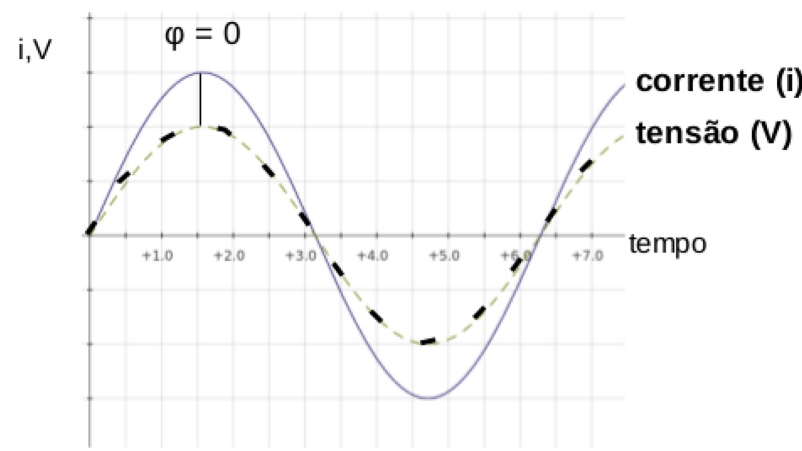

$3 a$

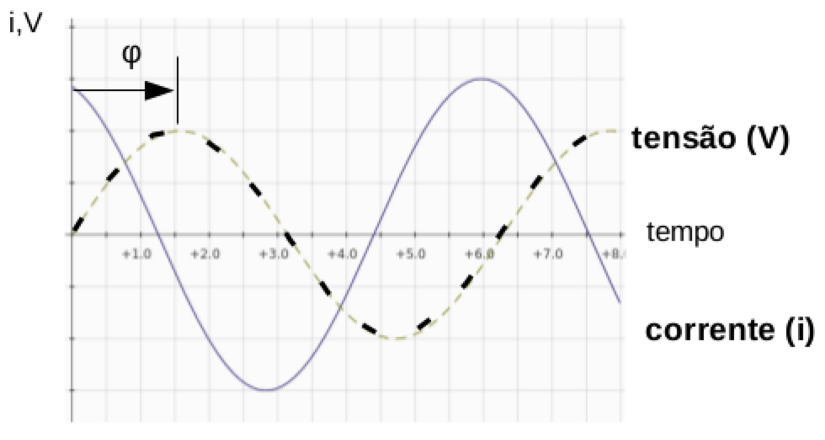

$3 b$

Figura 3: (a) Tensão e corrente auferidas no resistor, não há diferença de fase; (b) Tensão e corrente auferidas no capacitor, surge uma diferença de fase $\varphi$.

$$
\varphi=\arctan \left(\frac{\chi_{c}}{R}\right)
$$

A AIBC pode ser conduzida de diferentes maneiras. Pode ser empregada uma única frequência ou várias (método mono ou multifrequencial); podem ser feitas medidas de corpo inteiro ou de seus segmentos (pernas, braços e tronco); podem ser considerados vários modelos eletrofisiológicos para o corpo humano (como os representados nos circuitos da Figura 2). Cada modalidade de AIBC apresenta vantagens e desvantagens que precisam ser consideradas, pois podem reverter em resultados aceitáveis ou não dependendo dos objetivos da avaliação clínica 9] 10. A forma mais comum de AIBC, emprega medida de corpo inteiro e considera uma analogia do corpo humano ao circuito da Figura 2a percorrido por uma corrente de frequência fixa de $50 \mathrm{kHz}$ (monofrequencial) [9] [10. Esta modalidade de AIBC pode ser aplicada somente às pessoas saudáveis em estado normal de hidratação e permite determinar parâmetros como massa de gordura (MG), massa livre de gordura (MLG) e quantidade de água corporal total (ACT), não sendo apropriada todavia para a determinação dos parâmetros como a água extracelular (AEC) e da água intracelular (AIC) [2]. Estes últimos parâmetros podem ser melhor auferidos através de métodos multifrequenciais 2$][7]$ [1].

A Figura 4a mostra uma curva de bioimpedância em espectro de frequências obtida experimentalmente. O 
valor de $Z_{c}$ é maior em baixas frequências e diminui com o aumento desta 12 .

Observa-se que o circuito da Figura 2a não descreve apropriadamente esse comportamento eletrofisiológico que pode ser melhor aproximado pelo que é representado na Figura 2b. Quando este circuito (2b) é atravessado por uma corrente de baixa frequência, o capacitor praticamente bloqueia sua passagem pelo seu ramo superior forçando que circule pelo ramo inferior. Por isso, em baixas frequências, a impedância tende ao valor de $R_{A E C}$. No outro extremo, de altas frequências, o capacitor comporta-se como estando em curto e a impedância tende ao valor da resistência equivalente da associação em paralelo de $R_{A E C}$ e $R_{A I C}$ (Figura 4b).Resumindo, no corpo humano, correntes alternadas de baixa frequência carregam o "capacitor" biológico, favorecendo a condução pelo percurso extracelular, através da AEC, refletindo dessa forma somente esse espaço. Em frequências mais altas, superiores a $1 \mathrm{kHz}$ [12], a corrente consegue penetrar totalmente as membranas celulares e fluir também pela água intracelular (AIC), estabelecendo-se forte correlação da impedância com a quantidade de água total (ACT) [5] 12].

Para facilitar as análises, o circuito da Figura $2 \mathrm{~b}$ pode ser reduzido ao da Figura 2a através das relações (4) e (5), onde $R_{e q}$ e $\chi_{e q}$ passam a representar respectivamente $R$ e $\chi_{c}$ da equação (1), que por sua vez, teoricamente, tornam-se funções de $R_{A E C}, R_{A I C}$ e $\chi$.

$$
\begin{gathered}
R_{e q}=\frac{R_{A E C} \cdot R_{A I C} \cdot\left(R_{A E C}+R_{A I C}\right)+R_{A E C} \cdot \chi^{2}}{\left(R_{A E C}+R_{A I C}\right)^{2}+\chi^{2}} \\
\chi_{e q}=\frac{R_{A E C}^{2} \cdot \chi}{\left(R_{A E C}+R_{A I C}\right)^{2}+\chi^{2}}
\end{gathered}
$$

Onde

$$
\chi=\frac{1}{2 . \pi \cdot \text { frequência.C }}
$$

Este artigo propõe um experimento no qual sejam feitas medidas em corpo inteiro usando frequência en- tre $5 \mathrm{kHz}$ e $80 \mathrm{kHz}$, assumindo-se uma similitude entre os comportamentos eletrofisiológico do corpo humano e do circuito em série da Figura 2b. Esta escolha se justifica porque esse é o circuito mais simples que representa adequadamente o comportamento da bioimpedância medida através do método multifrequencial, posto que o modelo representado na Figura 2a é apropriado quando se adota o método monofrequencial, que usualmente emprega frequência de $50 \mathrm{kHz}$. Os valores de $Z_{c}$ e $\varphi$ podem ser obtidos do experimento que será descrito na subseção 2.2. A partir do sistema formado pelas equações (1) e (3) é possível determinar os valores de $R$ e $\chi_{c}$, os quais devem ser aplicados, juntamente com outros parâmetros antropométricos, às equações obtidas de regressões multivariadas, validadas contra métodos de referência 11 para a determinação das variáveis de interesse clínico. A acurácia dos modelos aumenta com a inclusão de informações como idade, altura, peso corporal e sexo, e isso acaba por subordinar a sensibilidade do uso das regressões à subpopulações específicas [5] [14].

O modelo de regressão adotado para a determinação da massa livre de gordura (MLG) foi o de Kyle 15], dado pela expressão (7), que utiliza os parâmetros $R$ e $\chi_{c}$ medidos a $50 \mathrm{kHz}$. Esse se presta muito bem para ilustrar as limitações que modelos desse tipo possuem. No caso particular, presta-se a AIBC de suíços, entre 22 e 94 anos, com índices de massa corporal entre 17,0 e $33,8 \mathrm{~kg} / \mathrm{m}^{2}$.

$$
\begin{aligned}
M L G & =-4,104+\left(\frac{0,518 \cdot(\text { altura })^{2}}{R_{(50 k H z)}}\right)+0,231 . \text { peso } \\
& +0,130 \cdot \chi_{c(50 k H z)}+4,229 . \text { fator }
\end{aligned}
$$

onde fator é 1 para homens e nulo para mulheres, a altura é medida em centímetros, o peso em quilogramas, $R$ e $\chi_{c}$ em ohms.

${ }^{1}$ Raios-X de dupla energia, densitometria, métodos antropométricos, diluição de isótopos 7]. Ver 13.

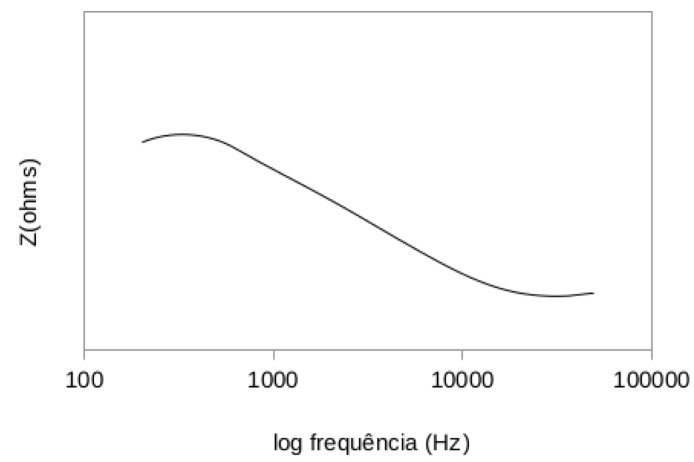

$4 a$

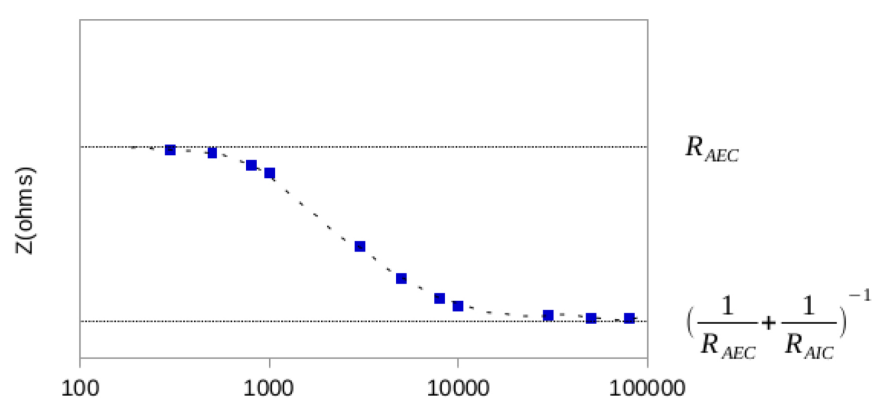

$\log$ frequência $(\mathrm{Hz})$

Figura 4: (a) Comportamento da bioimpedância em função da frequência da corrente alternada. (b) Impedância em função da frequência em um circuito como o representado na Figura $2 b$. 
$\mathrm{Na}$ realidade, muitos modelos como o de Kyle podem ser encontrados na literatura [7]. O emprego de um ou outro deve observar criteriosamente as premissas e a amostra populacional considerada, bem como o tipo/modelo de equipamento (comercial) utilizado porque esses medidores não seguem nenhuma padronização, utilizam diferentes modelos eletrofisiológicos e regressões que muitas vezes não são revelados [14] [16, levando a necessidade de validação desses dispositivos para uso através de outras técnicas [17]. A opção pelo modelo de Kyle, portanto, se deu por finalidade didática, para exemplificar como $R$ e $\chi_{c}$ são utilizados na determinação da composição corporal.

Obtida a MLG a partir de um modelo de regressão validado para a população de interesse, considerando-se que as medidas estejam sendo feitas em pessoas saudáveis com estado normal de hidratação, pode-se utilizar as equações (8), (9) e (10) para o cálculo de ACT , MG, $\% \mathrm{MG}$.

$$
\begin{aligned}
& A C T=0,73 . M L G \\
& M G=\text { peso }-M L G \\
& \% M G=\frac{M G}{\text { peso }} .100
\end{aligned}
$$

\subsection{Arranjo Experimental}

Na Figura 5 é apresentado um esquema do arranjo experimental. O resistor de referência $\left(R_{\text {ref }}\right)$ associado possui duas funções: é em relação ao mesmo que é medida a diferença de fase $(\varphi)$ e é através de seu valor conhecido que se pode calcular a corrente que passa pelo circuito. De forma alternativa um multímetro pode ser empregado

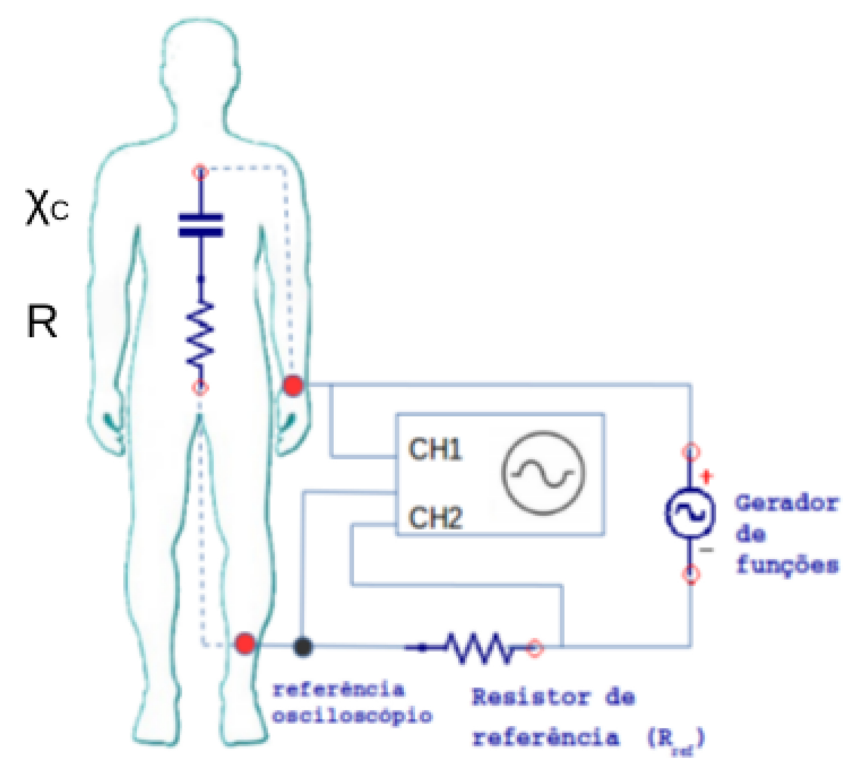

Figura 5: Esquema do arranjo experimental para medida de bioimpedância. para a medida direta da corrente, considerando que disponha da função true RMS (do inglês root mean square) para sinais alternados.

O osciloscópio fornece a diferença de potencial eficaz no resistor de referência $\left(V_{r}\right)$ e no corpo $\left(V_{c}\right)$, a frequência $(f)$ dos sinais e a diferença de tempo $(\Delta t)$ entre os picos dos sinais de cada um dos canais (CH1 corpo humano e $\mathrm{CH} 2$ resistor de referência). A diferença de fase $(\varphi)$ pode ser então calculada da relação $(11)$, a corrente $\left(I_{A C}\right)$, que passa por todo o sistema, da relação (12) e a impedância do corpo humano $\left(Z_{c}\right)$, da relação $(13)$.

$$
\begin{gathered}
\varphi=2 \pi f \Delta t \\
I_{A C}=\frac{V_{r}}{R_{r e f}} \\
Z_{c}=\frac{V_{c}}{I_{A C}}
\end{gathered}
$$

Substituindo (12) em (13):

$$
Z_{c}=\frac{V_{c} \cdot R_{r e f}}{V_{r}}
$$

Pode-se então resolver um sistema de equações com duas variáveis para se obter os valores de $\mathrm{R}$, e da reatância capacitiva $\left(\chi_{c}\right)$ do corpo humano, conforme equações (1) e (3).

Substituindo (3) em (1):

$$
R=Z_{c} \cdot \cos (\varphi)
$$

Finalmente substituindo a equação (15) na equação (3) pode-se calcular o valor $\chi_{c}$.

O acoplamento do circuito ao corpo humano pode ser feito através de eletrodos de ECG do tipo $\mathrm{Ag} / \mathrm{AgCl}$, molhados com gel condutor e conectados às partes internas do pulso e do tornozelo (Figura 6). Cabos tipo jacaréjacaré conectam os eletrodos de ECG à placa de ensaio ou matriz de contato (protoboard).

\section{Análise do Experimento}

\subsection{Medidas em circuito}

Inicialmente o circuito esquematizado na Figura 7 foi montado em uma placa de ensaio (protoboard) com o
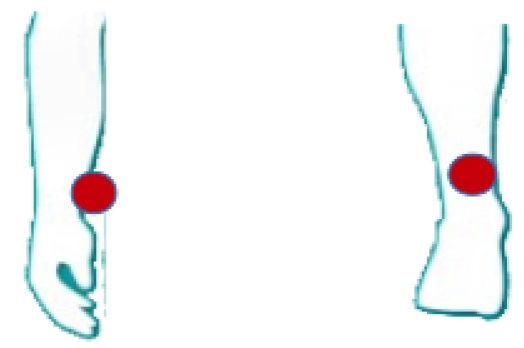

Figura 6: Posições de conexão dos eletrodos de ECG para medida de corpo inteiro. 


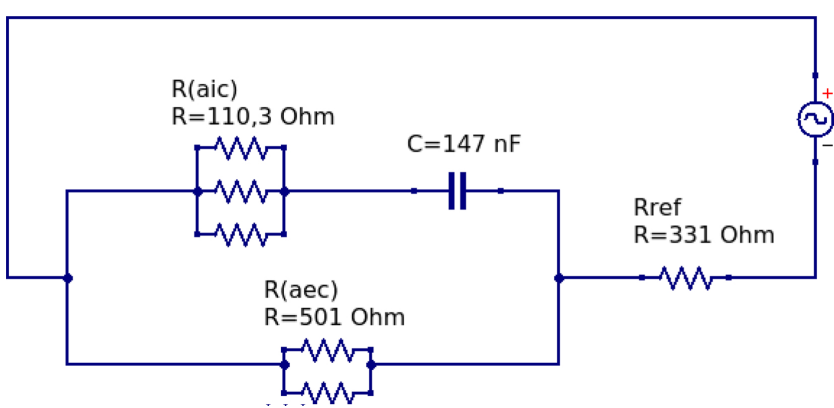

Figura 7: Circuito montado em protoboard para simular e validar o comportamento da bioimpedância em uma faixa de frequência de $500 \mathrm{~Hz}$ e $80 \mathrm{kHz}$. Os valores de resistência apresentados são valores equivalentes das associações em paralelo, medidos com multímetro (Tabela 1). A capacitância e o valor da resistência de referência também foram obtidos por medida direta com multímetro.

objetivo de simular e validar as medidas de bioimpedância no corpo humano quando a frequência varia entre $500 \mathrm{~Hz}$ e $80 \mathrm{kHz}$.

A $R_{A E C}$ foi simulada com a associação em paralelo de dois resistores de $1 k \Omega$ (valor nominal) e $R_{A I C}$ pela associação em paralelo de três resistores de $330 \Omega$ (valor nominal) . Os valores de resistência das associações, assim como do resistor de referência e a capacitância $(C)$ medidos diretamente com multímetro são apresentados na Tabela 1.

Submetendo-se o circuito à correntes alternadas de diferentes frequências, mediu-se os valores de $V_{r}, V_{c}$ e $\Delta t$, e pode-se calcular os valores de $\varphi, I, Z, R$ e $\chi_{c}$ conforme explicado na subseção 2.2. Os valores de $R_{e q}$ e $\chi_{e q} \mathrm{pu}-$ deram também ser calculados através das equações (4), (5), (6) e dos valores da Tabela 1. A seguir, as Figuras 8 e 9 apresentam, respectivamente, os comparativos entre os valores de $R$ e $R_{e q}$ e os valores de $\chi_{c}$ e $\chi_{e q}$ em função da frequência. Para facilitar a visualização do comportamento das grandezas, foram traçadas linhas guia para cada conjunto de pontos.

As figuras mostram que $R$ e $\chi_{c}$ calculados pelo método descrito na subseção 2.2 a partir de medidas experimentais, podem ser utilizados para se obter os valores de $R_{A E C}, R_{A I C}$ e $C$, uma vez que $R=R_{e q}$ e $\chi_{c}=\chi_{e q}$.

No circuito, os comportamentos de $\chi_{c}, Z, R$ e $\varphi$ em função da frequência pode ser observados na Figura 10. Em baixas frequências, a impedância $Z$ tende a $500 \Omega$, ou seja, à resistência $R$ que por sua vez tende ao valor

Tabela 1: Valores de $R_{A I C}, R_{A E C}, R_{r e f}$ e $C$ medidos diretamente com multímetro.

\begin{tabular}{lc}
\hline Componente do circuito & Valor medido \\
\hline$R_{A I C}(\Omega)$ & $110,3 \pm 1,4$ \\
$R_{A E C}(\Omega)$ & $501 \pm 9$ \\
$R_{\text {ref }}(\Omega)$ & $331 \pm 8$ \\
$C(n F)$ & $147 \pm 6$ \\
\hline
\end{tabular}

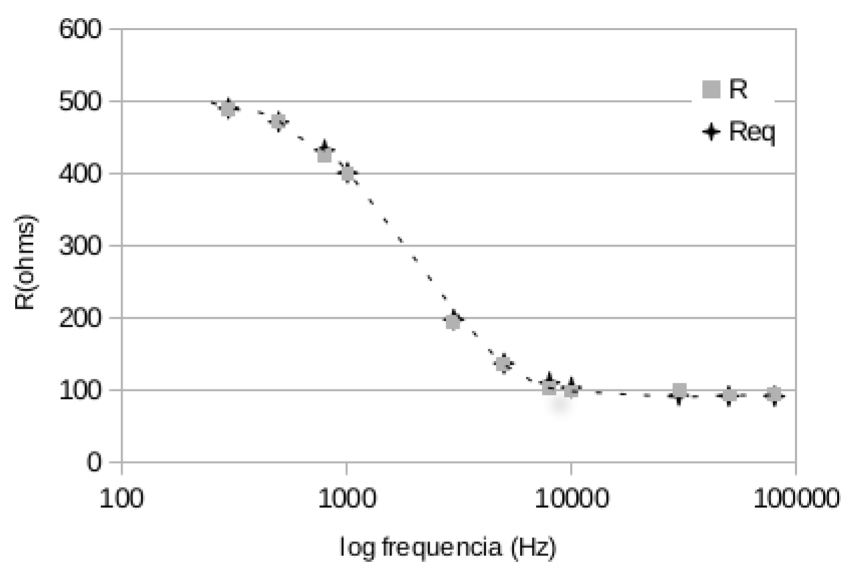

Figura 8: Comparativo entre valores de $R$ e $R_{e q}$ em função da frequência.

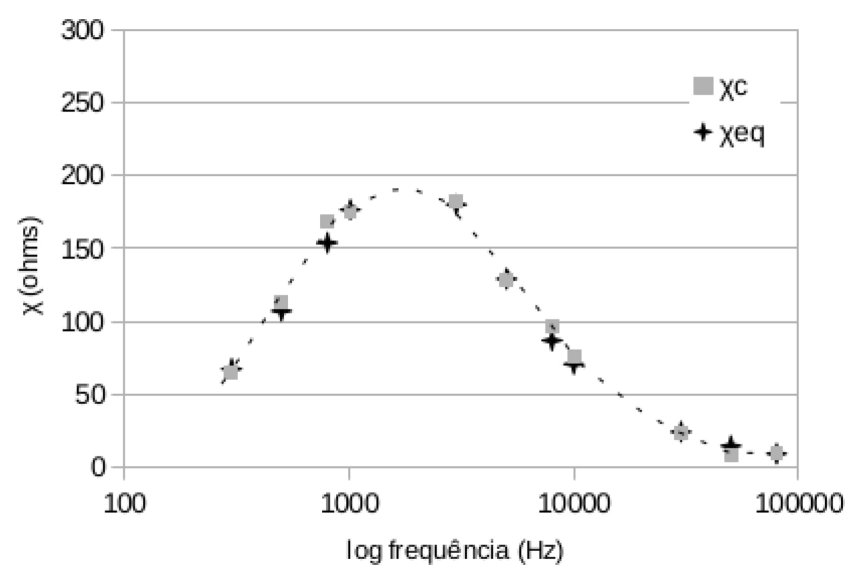

Figura 9: Comparativo entre valores de $\chi$ e $\chi_{e q}$ em função da frequência.

de $R_{A E C}$. Em altas frequências, a impedância também tende ao valor $\mathrm{R}=(94 \pm 8) \Omega$ que, nesse outro extremo, corresponde à associação em paralelo das resistências $R_{A E C}$ e $R_{A I C}$, ou seja, $(91,0 \pm 1,0) \Omega$. Quanto à reatância $\chi_{c}$, esta tende a zero em ambos os extremos de frequência como mostra a Figura 10, o que pode ser também verificado quando os limites da equação 5 são calculados para frequência tendendo a $\pm \infty$.

Os gráficos das Figuras 8 e 9 podem ser reunidos no diagrama de Cole-Cole [18], que relaciona o valor da reatância em função do valor da resistência. O semicírculo representa todos os pontos $\left(R_{e q}, \chi_{e q}\right)$ cujos valores podem ser calculados a partir das equações(4),(5), (6) e dos valores da Tabela 1. Os pontos da curva $R$ versus $\chi_{c}$ são os pontos experimentais, medidos de acordo com as instruções da subseção 2.2, com suas respectivas barras de erro. O tamanho do vetor do ponto origem até um ponto escolhido representa a impedância e o ângulo $\varphi$ representa a diferença de fase. Cada ponto está associado a uma frequência que aumenta da direita para a esquerda na figura. No limite de baixa frequência o semicírculo se 


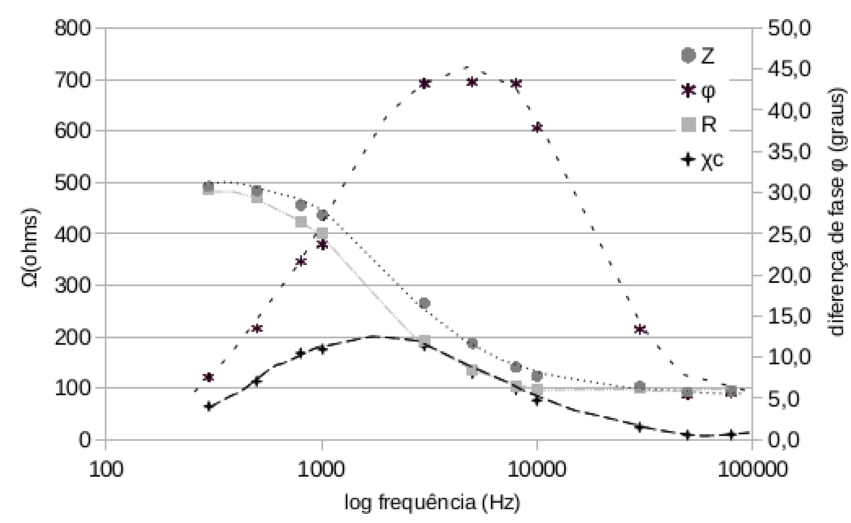

Figura 10: Valores de $Z, R, \varphi, \chi_{c}$ em função da frequência, obtidos pelo método descrito na subseção 2.2 aplicado ao circuito da Figura 7.

aproxima do valor de $R_{A E C}$ e no de alta frequência ao valor da associação em paralelo de $R_{A E C}$ e $R_{A I C}$.

A Figura 12 é o diagrama de Cole-Cole $[18$ construído com dados extraídos de um circuito idêntico ao da Figura 7 exceto pelo fato do capacitor de $(147 \pm 6) \mathrm{nF}$ ter sido substituído por outro de $(11,7 \pm 0,5)$ nF. Observa-se comparando-se as Figuras 11 e 12 que a frequência em que ocorre a maior diferença de fase aumenta, como se os pontos $\left(R_{e q}, \chi_{e q}\right)$ se "deslocassem para a direita" sobre o semicírculo, determinado pelos valores de $R_{A E C}$ e $R_{A I C}$. Portanto, qualquer mudança da capacitância do circuito terá consequência na frequência em que ocorrerá maior impedância $Z$ ou diferença de fase $\varphi$.

\subsection{Medidas "in vivo"}

Com o arranjo da Figura 5, empregando os eletrodos de ECG conectados a porção interna do pulso e tornozelo de um voluntário, mediu-se a bioimpedância variando-se a frequência de 5 a $80 \mathrm{kHz}$. O emprego de frequências não inferiores a $5 \mathrm{kHz}$ é necessário para se evitar que as medidas sejam distorcidas pelo efeito de interface peleeletrodo. Conforme publicado por SAADI H. e ATTARI M. [19], esse efeito começa a ser significativo abaixo de $1 \mathrm{kHz}$, mas no arranjo experimental proposto nesse trabalho (Figura 3), medidas abaixo de $5 \mathrm{kHz}$ mostraram muito ruído e consequentemente grandes incertezas. Por isso, a medida a $1 \mathrm{kHz}$ serviu somente para estimação do $R_{A E C}$ optando-se posteriormente por só considerar, para avaliação de bioimpedância, as medidas feitas com frequência superior a $5 \mathrm{kHz}$.

Nas medidas em vivo, o ideal é empregar correntes menores que $1 \mathrm{~mA}$. Mas com os recursos disponíveis no osciloscópio, como a possibilidade de pausar a tomada do sinal e anotar ou extrair posteriormente as medidas, é possível tomar os dados minimizando-se ao máximo o tempo que a corrente passa pelo corpo, o que possibilita, em algumas condições, utilizar-se de correntes maiores, mas sempre inferiores a $4 \mathrm{~mA}$.

A Figura 13 mostra os valores de bioimpedância $\left(Z_{c}\right)$ e ângulo de diferença de fase $(\varphi)$ em função do logaritmo da frequência, e também os valores de $R$ e $\chi_{c}$ calculados de acordo com o método da subseção 2.2 para medidas "in vivo".

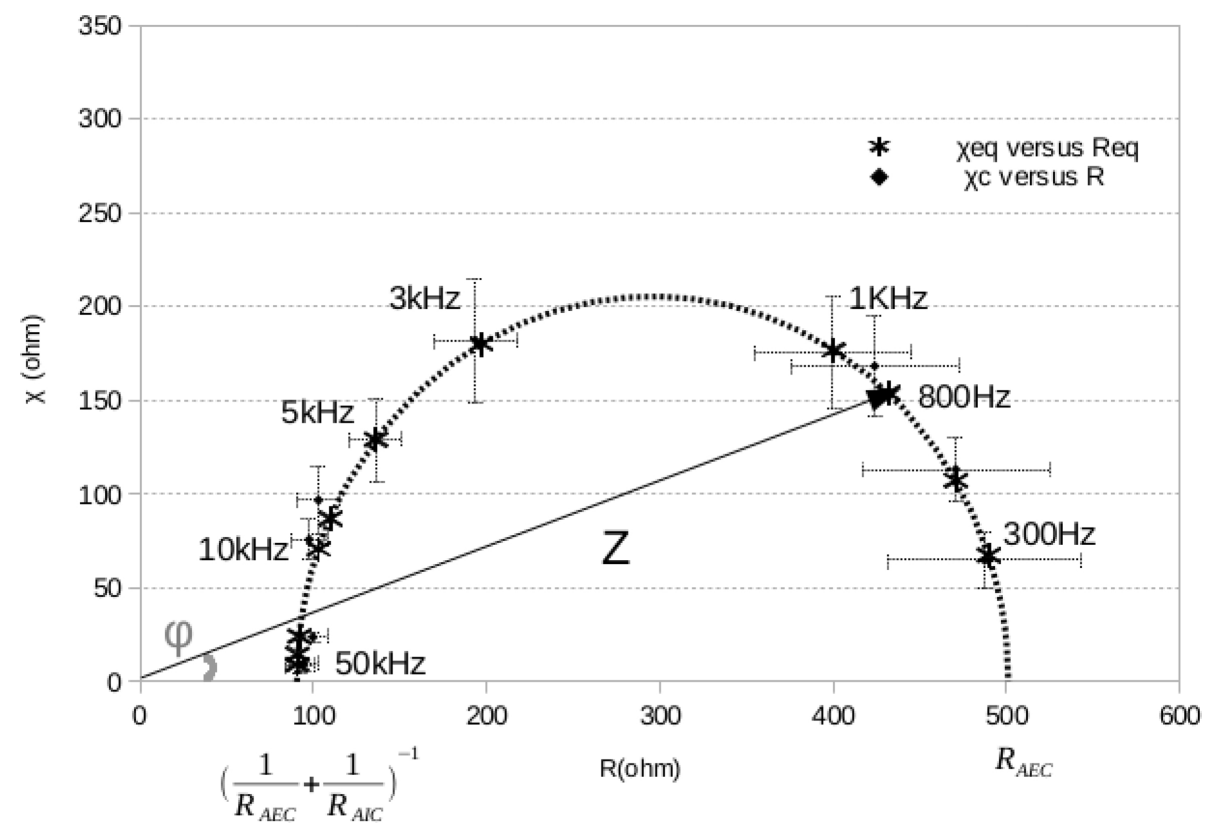

Figura 11: Diagrama de Cole-Cole representando dados das Figuras 8 e 9 . Nessa representação o tamanho do vetor $Z$ representa a impedância e $\varphi$ a diferença de fase a $800 \mathrm{~Hz}$ de um ponto $\left(R_{e q}, \chi_{e q}\right)$ cujos valores foram calculados a partir das equações (4),(5),(6) e dos valores da Tabela 1 . Os pontos $\left(R, \chi_{c}\right)$ obtidos experimentalmente estão representados com suas respectivas barras de erro. $O$ semicírculo representa todos os pontos $\left(R_{e q}, \chi_{e q}\right)$ possíveis do modelo eletrofisiológico escolhido que emprega um determinado valor de $R_{A E C}$ e de $R_{A I C}$. 


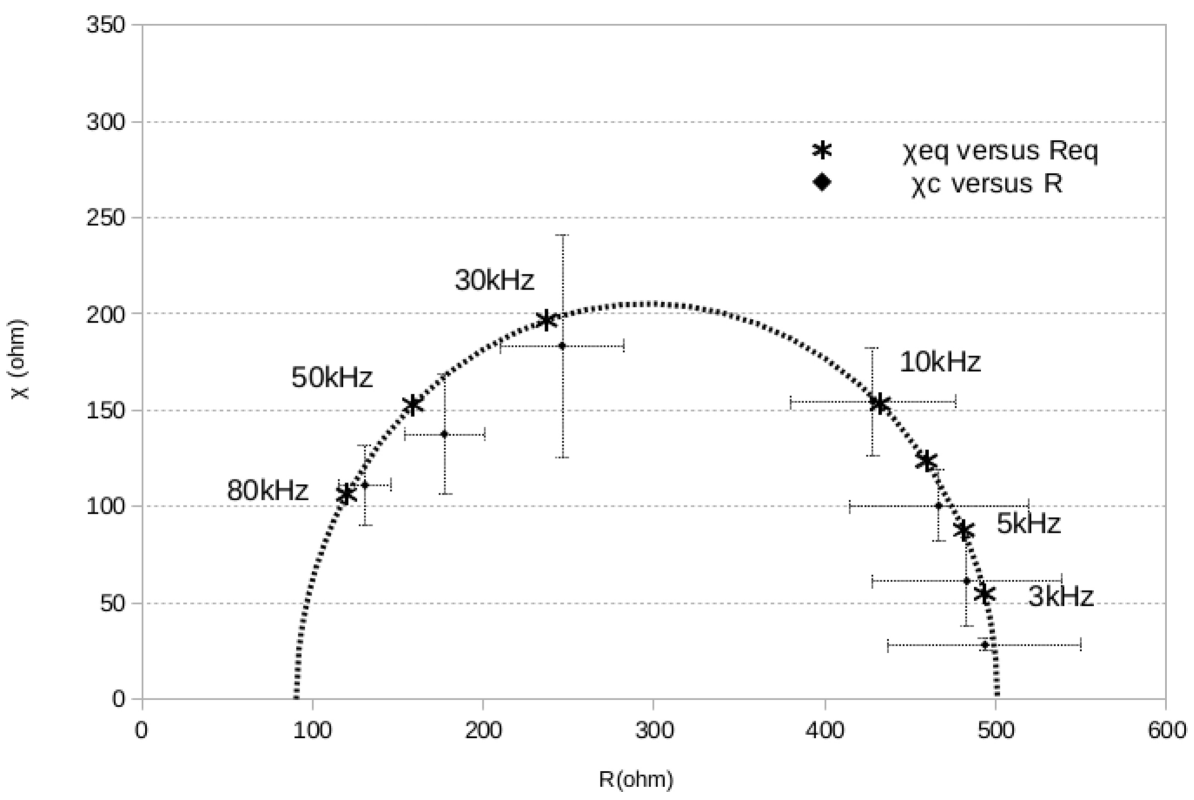

Figura 12: Diagrama de Cole-Cole construído com dados extraídos de um circuito idêntico ao da Figura 7 , mas com capacitor de $(147 \pm 6) \mathrm{nF}$ substituído por outro de $(11,7 \pm 0,5) \mathrm{nF}$. O semicírculo representa todos os pontos $\left(R_{e q}, \chi_{e q}\right)$ determinados pelos valores de $R_{A E C}$ e de $R_{A I C}$, de acordo com o modelo eletrofisiológico escolhido para a bioimpedância.

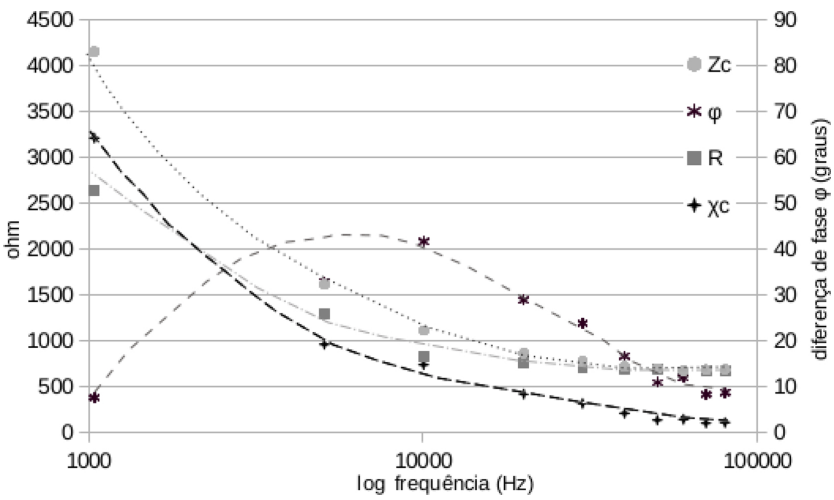

Figura 13: Valores de $Z_{c}, \varphi$ em função do logaritmo da frequência, e também os valores de $R$ e $\chi_{c}$ calculados de acordo com o método da subseção 2.2 .

Para verificarmos se o circuito da Figura $2 \mathrm{~b}$ pode simular eletrofisiologicamente o corpo humano, é necessário extrair da Figura 13 os valores de $R_{A E C}$ e $R_{A I C}$. A 1 $\mathrm{kHz}, R$ vale $(2,63 \pm 0,52) k \Omega$, valor que será assumido como $R_{A E C}$. Em $80 \mathrm{kHz}, R$ tende a $(674 \pm 65) \Omega$ e por isso o valor de $R_{A I C}$ tende à $(0,91 \pm 0,12) k \Omega$. O conjunto de pontos $\left(R_{e q}, \chi_{e q}\right)$ possíveis - calculados a partir das equações (4),(5) e (6) e que em conjunto formam o semicírculo no diagrama da Figura 14 - foram obtidos a partir de um melhor ajuste, para o qual o valor da capacitância $(C)$ foi definido em $11,5 \mathrm{nF}$.

A Figura 14 mostra, a partir dos dados coletados, que o semicírculo da Figura 2b é adequado para a representação do comportamento eletrofisiológico do corpo humano para frequências acima de $5 \mathrm{kHz}$. Diferentemente do que ocorre nos equipamentos comerciais que utilizam método monofrequencial a $50 \mathrm{kHz}$, onde a maior diferença de fase ocorre nessa freqüência, a diferença de fase máxima no experimento ocorreu em frequência inferior a $5 \mathrm{kHz}$. Baseado no que foi discutido a partir da Figura 12 , especula-se que algum efeito capacitivo adicional tenha ocorrido, provavelmente causado pelos eletrodos em contato com a pele. Desta forma, é possível estimar qual seria a capacitância, descontada dos efeitos dos eletrodos, para o qual a diferença de fase é máxima a $50 \mathrm{kHz}$. Esse valor, que representa a capacitância equivalente do corpo humano quando este é reduzido ao modelo da Figura $2 \mathrm{~b}$, é de aproximadamente $1,6 \mathrm{nF}$.

O arranjo proposto na Figura 5 foi então empregado em uma medida a $50 \mathrm{kHz}$ em um voluntário com o objetivo de reproduzir o exame clínico monofrequencial de bioimpedância para a determinação da composição corporal. Hipoteticamente considerou-se que essa medida tenha sido feita em um cidadão suíço, com índices de massa corporal de $26,8 \mathrm{~kg} / \mathrm{m}^{2}$ (altura de $178 \mathrm{~cm}$ e peso corporal de $85 \mathrm{Kg}$ ) para ser possível o uso da equação (7). Os valores calculados são apresentados na Tabela 2.

O modelo de Kyle 15 estabelece algumas relações que são válidas independentemente do modelo de regressão que se adote. Uma delas é que a MLG é diretamente proporcional à quantidade de água total (ACT) que por sua vez é inversamente proporcional à $\mathrm{R}$ [20]. A outra é que a MLG cresce com $\chi_{c}$, grandeza que é diretamente proporcional à relação AIC/AEC [5] e desta forma reflete o aumento de massa muscular, uma vez que AIC aumenta com a constituição dos músculos.

Como discutido na seção 2.1, o uso de quaisquer modelos requer sempre algum tipo de validação, seja por conta das características específicas da subpopulação que 


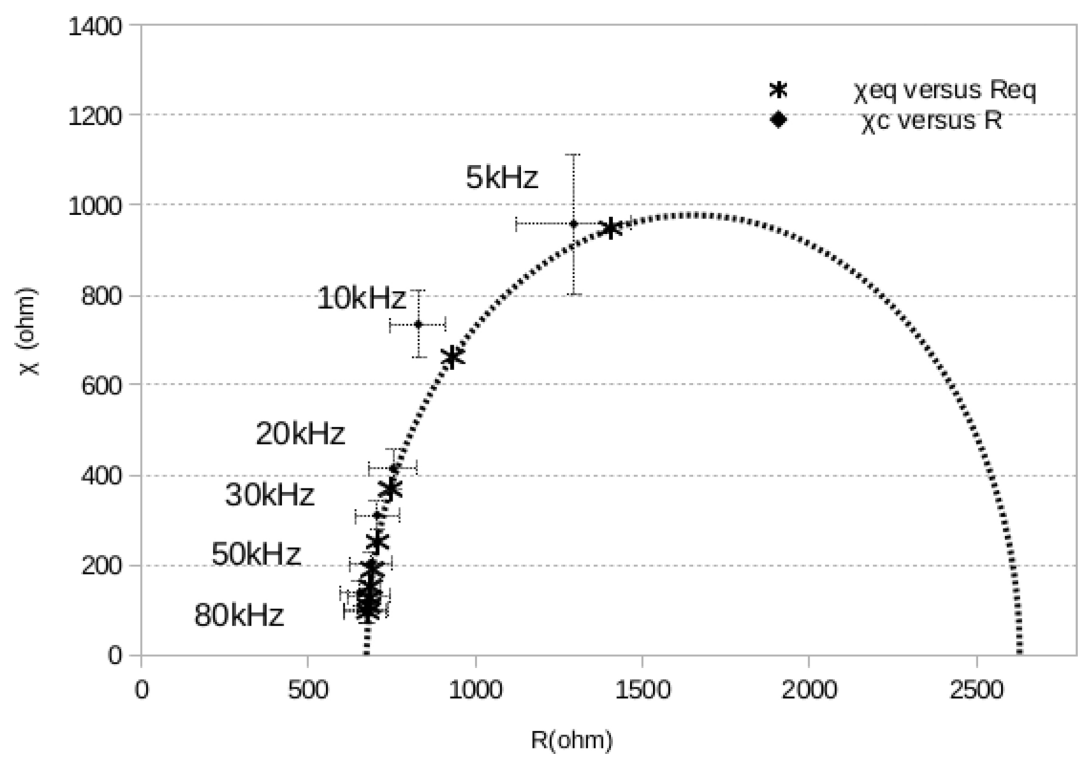

Figura 14: Diagrama de Cole-Cole para medida de impedância "in vivo" com o arranjo experimental proposto quando se infere os valores de $R_{A I C}=0,91 \pm 0,12 \mathrm{k} \Omega$ e $R_{A E C}=674 \pm 65 \mathrm{k} \Omega$ da Figura 13 e define-se um valor ideal para $C=11,5 \mathrm{nF}$.

Tabela 2: Valores calculados de MLG, ACT, MG, \%MG, segundo as equações (7), (8), (9) e (10), supondo-se que a medida tenha sido feita um cidadão suíço, do sexo masculino, com índices de massa corporal de $26,8 \mathrm{~kg} / \mathrm{m}^{2}$, altura de $178 \mathrm{~cm}$ e peso corporal de $85 \mathrm{~kg}$.

\begin{tabular}{lccccc}
\hline$R(\Omega)$ & $\chi_{c}(\Omega)$ & $\mathrm{MLG}(\mathrm{kg})$ & $\mathrm{ACT}(\mathrm{kg})$ & $\mathrm{MG}(\mathrm{kg})$ & $\% \mathrm{MG}$ \\
\hline $682 \pm 64$ & $130 \pm 18$ & $56,5 \pm 3,3$ & $41,2 \pm 2,4$ & $28,5 \pm 3,3$ & 29,7 a 37,4 \\
\hline
\end{tabular}

serviu de referência para construção das regressões, seja pelo tipo de equipamento de AIBC que fora empregado. Equipamentos diferentes podem medir valores de $R$ e $\chi_{c}$ diferentes, ou por conta de características específicas de sua construção ou por empregarem modelos eletrofisiológicos distintos, os quais geralmente não são divulgados pelos fabricantes. Dessa forma, para as avaliações clínicas de bioimpedância recomenda-se pesquisa detalhada sobre como são feitos os cálculos, quais modelos eletrofisiológicos e de regressão foram adotados e não se pode desconsiderar uma avaliação da validade da regressão à subpopulação que será submetida ao procedimento clínico. Pode ser necessária a realização de testes de aderência ou mesmo a obtenção de mais informações junto ao fabricante do equipamento. Medidas podem ser feitas com diferentes valores de freqüências, o que implicará em diferenças de fase menores ou maiores, desde que existam, para um determinado valor escolhido, equações de regressão correspondentes a cada subpopulação, de preferência construídas com um mesmo tipo de equipamento.

\section{Uso didático}

Do ponto de vista didático-experimental, a proposta apresentada pretende demonstrar aos estudantes da área da saúde como um circuito elétrico simples do tipo RC, em corrente alternada, pode simular as propriedades bioelétricas do corpo humano, permitindo compreender o princípio de funcionamento dos equipamentos comerciais normalmente empregados na AIBC. Esta proposta foi desenvolvida a partir da experiência dos autores no grupo do Programa de Educação Tutorial (PET) do Ministério de Educação - PET Tecnologias em Saúde, cujo principal objetivo é contribuir para o enfrentamento do desafio de ensinar, discutir, refletir e divulgar temas ligados às áreas tradicionais do conhecimento como Física, Química, Matemática e Computação entre estudantes de graduação da área da Saúde e a comunidade, usando como temática o funcionamento do corpo humano.

Para a execução do experimento são utilizados equipamentos normalmente encontrados em laboratórios dos cursos de graduação ou mesmo em centros de formação ou departamentos / setores de apoio da área da saúde: osciloscópio de $70 \mathrm{MHz}$ ou mais, com dois canais, gerador de funções senoidais, com seletor de frequências entre $100 \mathrm{~Hz}$ e $100 \mathrm{KHz}$, multímetro digital, placa de ensaio ou matriz de contato (protoboard), resistores de precisão (com 1\% de tolerância no valor nominal) de $330 \Omega$ e $1000 \Omega$, capacitores cerâmicos com valores nominais entre $10 \mathrm{nF}$ e 150nF, cabos para conexões, eletrodos descartáveis de eletrocardiograma (ECG) e gel condutor para medidas "in-vivo".

Sugere-se que o uso didático do experimento seja feito em três etapas. A primeira deve permitir que os estudantes familiarizem-se com o uso do osciloscópio e demais equipamentos, montem o circuito equivalente para simular as medidas de bioimpedância e façam os cálculos para 
comparação das grandezas físicas medidas e calculadas. Os estudantes devem ser incumbidos de reproduzir as medidas e os gráficos da subseção 3.1 para um único valor de capacitor, realizando as medidas no circuito equivalente, com valores de resistências e capacitor conhecidos. O funcionamento de um circuito $\mathrm{RC}$ em corrente alternada e a analogia dos componentes empregados na simulação com as propriedades elétricas do corpo humano devem ser explorados nesta etapa. A dependência da impedância e variação do ângulo de fase com a frequência deve permitir a introdução do princípio de funcionamento dos equipamentos de AIBC.

Em seguida, numa segunda etapa, os estudantes devem conduzir medidas "in vivo" em um integrante voluntário do grupo, observando as condições de segurança estabelecidas. As medidas devem ser realizadas em baixa frequência $(1 \mathrm{kHz})$ e na faixa de $5 \mathrm{kHz}$ a $80 \mathrm{kHz}$, relacionando o comportamento das curvas de bioimpedância $\left(Z_{c}, R, \chi_{c}, \varphi\right)$ com aquele obtido anteriormente, no circuito da etapa anterior. Assim podem estimar os valores de $R_{A E C}, R_{A I C}$ e $C$, e avaliar o circuito da Figura $2 \mathrm{~b}$ como um modelo eletrofisiológico adequado para a medida de bioimpedância efetiva. Aqui cabe uma discussão sobre o valor máximo da diferença de fase encontrado e a escolha da frequência normalmente empregada nos equipamentos comerciais do tipo monofrequencial.

$\mathrm{Na}$ etapa final, os valores determinados experimentalmente de $R$ e $\chi_{c}$ para $50 \mathrm{kHz}$, juntamente com parâmetros antropométricos do voluntário, devem ser empregados no cálculo das variáveis de interesse clínico (MLG, ACT, MG e \%MG), de acordo com o modelo de Kyle 15] e das equações (8), (9) e (10), comparando, quando possível, com os resultados obtidos com um equipamento comercial de AIBC. Importante é conduzir uma discussão sobre as limitações e possíveis problemas que o uso não crítico dos equipamentos de medidas comerciais pode acarretar, ressaltando-se a necessidade de se verificar como os cálculos são feitos, como foram calibrados os equipamentos e se estes se prestam à avaliação de composição corporal de uma determinada subpopulação. Para concluir, pode-se discutir com os estudantes outros métodos de avaliação corporal, destacando suas vantagens e desvantagens em relação à bioimpedância.

\section{Conclusões}

Nesse artigo foi apresentado um experimento para o ensino dos fundamentos de bioimpedância para estudantes da área da saúde, empregando um circuito do tipo $\mathrm{RC}$ para simular as propriedades bioelétricas do corpo humano. A estratégia de abordar de forma prática e contextualizada os conceitos, associando-os com uma aplicação clínica, busca facilitar a assimilação de tópicos particularmente difíceis de serem compreendidos por esse público através da forma tradicional de ensino. Adicionalmente pode ser feita uma discussão sobre o uso de modelos para a aquisição de informações sobre a composição corporal, da qual se ressalta a limitação de suas aplicações à subpopulações específicas, a partir das quais são originados os dados para as curvas de regressão. O tipo de equipamento empregado e seu princípio físico de funcionamento também podem alterar os resultados das medidas por conta dos diferentes modelos fisiológicos e de regressão que os fabricantes adotam, informações que muitas vezes são omitidas e impactam diretamente na aplicação. Concluiu-se que a operacionalização do experimento para fins didáticos é viável e seu emprego adequado para que os estudantes ascendam melhor compreensão da medida de bioimpedância e tornem-se críticos em relação ao emprego de equipamentos comerciais na prática clínica. Por fim, a exposição prática e conceitual usada nesta proposta reforça a importância de se re-examinar o propósito do conhecimento tradicional disciplinar na solução de problemas, muitas vezes pragmáticos, da área da saúde.

\section{Referências}

[1] C.R. de Mattos e A.V.N. Drummond, Cad. Bras. Ensino Física. 21, 1 (2004).

[2] M.S. Mialich, J.F. Sicchieri e A.A.J. Junior, Int J Clin Nutr. 2, 1 (2014).

[3] R. Parra, R. Nahon, D. Braga, N. Medeiros, A.V. Pino e M.N. Souza, in Anais XXIV Congresso Brasileiro de Engenharia Biomédica-CBED, Uberlândia, 2014 (Sociedade Brasileira de Engenharia Biomédica, Rioi de Janeiro, 2014), p. 961.

[4] W.C. Chumlea, Int. J .Obes. 26, 12 (2002).

[5] R.F. Kushner, J Am Coll Nutr. 11, 2 (1992).

[6] F.W.A. Sears e M.W.A. Zemansky, Física. (Ao Livro Técnico, Rio de Janeiro, 1971), v.3, p. 804.

[7] U.G. Kyle, Clin.Nutr, 23, 5 (2004).

[8] S.J. Carlson-Newberry e R.B. Costello, Emerging Technologies for Nutrition Research (National Academy Press, Washington, 1997), v. 1, p. 173.

[9] H.C. Lukaski, Am. J. Clin. Nutr. 64, 3 (1996).

[10] R. Gudivaka, D.A. Schoeller, R.F. Kushner e M.J.G. Bolt, J. Appl. Physiol. 87, 3 (1999).

[11] P. Deurenberg, A. Tagliabue e F.J.Schouten, Br. J. Nutr. 73, 3 (1995).

[12] E.C. Hoffer, C.K. Meador e D.C. Simpson, J. Appl. Physiol. 27, 4 (1969).

[13] D.M. Sigulem, M.U. Devincenzi e A.C. Lessa, J Pediatr. 76, 3 (2000).

[14] D.A. Schoeller, Nutrition. 12, 11 (1996).

[15] U.G. Kyle, L. Genton, L. Karsegard, D.O. Slosman e C. Pichard, Nutrition. 17, 3 (2001).

[16] S.Z. Yanovski, V.S. Hubbard, S.B. Heymsfield e H.C. Lukaski, Am. J. Clin. Nutr. 64, 3, (1996).

[17] L. Wang e S.S. Hui, BioMed Res.Int. 2015, 614858 (2015).

[18] K.S. Cole e R.H. Cole, J.Chem.Phys. 9, 4 (1941).

[19] H. Saadi e M. Attari, in Anais 2nd International Conference on Advances in Biomedical Engineering, Tripoli, 2013, editado por H. Amoud (Springer, Cham, 2014) p. $49-52$.

[20] H.C. Lukaski, P.E. Johnson, W.W. Bolonchuk e G.I. Lykken, Am. J. Clin. Nutr. 41, 4 (1985). 